[2] C. Neukomm, Le Lail, 1927 , p. 728 et 843.

[3] BREW JAMEs, cité dans le Bulletin mensuel des renseignements agricoles et des maladies des plantes, 1922, p. 1483;

\title{
LA MÉTHODE SYNTHÉTIQUE DANS L'ÉTUDE DU LAIT LE LAIT AU POINT DE VUE COLLOIDAL RECHERCHES SUR LE MÉCANISIME DE L'ACTION DE LA PRÉSURE
}

\author{
par Ch. PORCHER, \\ Docteur ès sciences physiques
}

(Suite)

\section{LE CASEINATE DE CALCIUM TEL QU'IL EXISTE DANS LE LAIT}

La caséine existe dans le lait de vache à l'état de caséinate de calcium, lequel est uni colloïdalement aux phosphates de calcium pour former un complexe. Mais par la pensée nous pouvons très bien distraire ces derniers sels, isoler par conséquent le caséinate de calcium, et se demander quel peut en être le $p \mathrm{H}$, autrement dit, quelle en est la richesse en chaux ? Sera-ce un easéinate acide, donc d'un $p \mathrm{H}<7$, un caséinate alcalin, d'un $p \mathrm{H}>7$, ou un caséinate neutre ou sensiblement neutre d'un $p \mathrm{H}=7$ ou voisin de 7 .

Il faut, tout de suite, dans le cas du lait de vache dont la réaction est toujours acide, éliminer la question d'un $p \mathrm{H}$ nettement alcalin. Restent done les hypothèses d'un $p \mathrm{H}$ acide et d'un $p \mathrm{H}$ neutre, c'est-àdire d'un caséinate aux environs de 600 , entre 590 et 610 , c'est-à-dire neutre ou fort voisin de la neutralité.

Lait et complexe sont des mélanges : leur réaction à l'un comme à l'autre est une somme. - Dans l'un comme dans l'autre cas, qu'il s'agisse de la liqueur aux multiples éléments qu'est le lait ou de la solution beaucoup plus simple du complexe, il y existe des éléments d'une acidité marquée ; ce sont les phosphates bi- et monopotassiques dans le cas du lait, le phosphate mono-calcique dans le cas du complexe. S'ils disparaissent, la partie qui reste tend vers l'alcalinité.

Des expériences de dialyse effectuées sur le lait ou le complexe, tel qu'il sera préparé plus tard, nous montrent en effet une marche non douteuse vers l'alcalinité de la liqueur restée dans le dialyseur.

Tel lait témoin dont le $p H$ est de $6,81,-p H$ élevé pour un lait, voit ce $p \mathrm{H}$ monter à 7,05 par dialyse ; dans tel autre, le $p \mathrm{H}$ passe de 6,98 . à 7,20 . Le $p H$ de tel complexe passe par dialyse de 6,91 à 7,12 .

Il n'y a pas lieu d'insister davantage ici sur les effets de la dialyse sur le lait et sur le complexe ; la place en est indiquée lorsque nous étudierons le mécanisme de l'emprésurage. Mais nous avons voulu retenir des quelques chiffres donnés que la dialyse a pour but de faire tendre 
la partie restée dans le dialyseur nettement vers l'alcalinité. Quelle en est, en substance, la composition chimique ? La réponse à cette question nous donnera probablement la raison de la modification du $p H$ dans le sens qui vient d'être indiqué. Dans le cas du lait, la partie restante est formée essentiellement d'un mélange de phosphates de calcium insolubles et de caséinate de caleium, car les chlorures, les phosphates alcalins, les citrates, le lactose ont traversé la paroi. Dans le cas du complexe, plus simple, avec lequel nous pouvons donc mieux suivre la marche du phénomène, le phosphate mono-calcique acide et le phosphate bicalcique ont disparu du mélange ; il restera dans le manchon du dialyseur le caséinate calcique et le phosphate tricalcique insoluble.

Le caséinate calcique naturel est neutre. - Nous sommes fondé à penser que le caséinate calcique en question est bien celui qui se trouvait à l'origine, soit dans le lait, soit dans le complexe, parce que, lorsqu'on fait dialyser une solution pure de caséinate de calcium de $p H<7$, rien ne passe ; c'est un colloïde parfait qui ne traverse pas les membranes serrées que nous employons. Il est alors logique de supposer que la réaction du liquide resté dans le dialyseur est celle du caséinate tel qu'il existe, soit dans le lait, soit dans le complexe. Or, cette réaction reste aux environs du $p \mathrm{H}=7$, et ne le dépasse jamais de beaucoup. Elle correspond à un caséinate à $600-610$, qui se trouve voisin de la neutralité ou très légèrement alcalin.

On voit que nous sommes arrivé à cette notion que le caséinate du lait devait être voisin de la neutralité par des considérations basées sur un phénomène physique : la dialyse. Nous verrons plus loin que nous y serons amené également par des considérations d'ordre chimique sur la répartition de la chaux entre la caséine et l'acide phosphorique dans le complexe.

Il est sans doute utile de citer encore un autre ordre de faits sur lesquels nous aurons à revenir et qui plaident dans le même sens que ceux qui précèdent.

Il répond au changement de réaction de la liqueur lors de l'emprésurage. Lorsqu'on emprésure un lait d'un $p H$ donné, le sérum est nettement plus acide. On a précipité par l'emprésurage, comme nous le verrons, le phosphate tri-calcique, presque tout le phosphate bi-calcique, et le paracaséinate de calcium, bref, des éléments neutres, et on a laissé dans la liqueur les éléments plus acides.

On voit tout l'intérêt qu'il y a à comparer ce que donnent chacun de leur côté la dialyse et l'emprésurage. Par la dialyse, on enlève la partie acide du lait et on laisse dans le dialyseur la partie la plus alcaline qui, en fait, reste voisine de la neutralité vraie : le complexe caséinate de caleium + phosphates de calcium. Par l'emprésurage, on précipite celle-ci et on laisse dans la liqueur les éléments les plus acides. Les 
données que l'on retire de l'un et de l'autre de ces processus si différents, lè premier, physique, le second, biochimique, sont concordantes.

Le caséinate calcique naturel n'est pas un mélange. - L. L. vaN, SLYKe et A. W. Bosworth estiment que le caséinate qui se trouve dans le lait serait du caséinate tétracalcique, c'est-à-dire octovalent. Or, un tel sel, fait remarquer Palmer (165) a un $p H$ élevé $>8$, et comme le $p \mathrm{H}$ du lait à l'état naturel oscille autour de $6,5-6,6$, on ne saurait dono admettre que ce caséinate y soit seul; vraisemblablement le caséinate du lait serait alors un mélange de caséinate tétracalcique, basique à la phtaléine du phénol, avec un caséinate plus acide.

Les considérations dans lesquelles nous sommes entré relativement à la saturation progressive de lá caséine par la chaux ne nous engagent pas à accepter une semblable manière de voir. Nous sommes porté à penser que le caséinate du lait, voisin de la neutralité vraie : $p \mathrm{H}=7$, est un sel défini ou si c'est un mélange, ce ne peut être qu'un mélange de caséinates très voisins par leur réaction.

LE CASEINATE CALCIQUE DU LAIT DANS SES RAPPORTS AVEC L'ACIDITE TITRABLE, - L'étude du caséinate calcique du lait va nous permettre d'expliquer à quoi est due, en partie du moins, l'acidité titrable du lait. Beaucoup de travaux ont été faits sur cette question. L'introduction dans la mesure de la réaction des liqueurs de l'organisme de la notion du $p \mathrm{H}$, autrement dit, de l'acidité actuelle, oblige à regarder de très près à quoi est dûe l'acidité potentielle du lait, l'ancienne notion que l'on mesure par les méthodes titrimétriques.

Dans un travail qui paraîtra un peu plus tard sur l'acidité du lait, nous dissèquerons celle-ci tant sous son aspect potentiel que sous son aspect actuel, et nous chercherons à déterminer quels en sont les facteurs, leur valeur relative, leur importance, leur signification.

A. TAPERNOtix (210), étudiant les laits de grands mélanges, s'est employé à montrer comment varient l'acidité actuelle et l'acidité potentielle dans diverses circonstances, et il a noté que souvent les oscillations de l'une et de l'autre étaient parallèles.

Si l'on fait porter les recherches sur des laits individuels, c'est alors que l'on jugera de la grande différence de ces deux données, l'une d'ailleurs d'essence chimique grossière, très artificielle, toute relative, puisque les chiffres qu'elle fournit varient a vec l'indicateur employé, l'autre d'essence physique, fournissant une valeur absolue. Néanmoins, la grande pratique de l'industrie laitière ayant recours à la titrimétrie dans l'immense majorité des cas, il importe de voir dans quelle mesure le caséinate de calcium joue sur le degré d'acidité trouvé. Qu'il s'agisse de degrés Soxhlet, de degrés Dornic, de degrés Thorner, etc..., c'est toujours au

(210) A. TAPERNoUx. - Les relations entre l'acidité potentielle et l'acidité actuelle du Iait. - Thèse Doct. Vét., Lyon, 3 mai 1928 , et Le Lait, 1928, 8, 686, 795 et 916. 
virage de la phénol-phtaléine que l'on s'arrête dans la détermination de l'acidité. Divers facteurs sont à considérer, mais ceux qui interviennent en premier lieu sont, d'une part, parmi les éléments solubles cristalloïdes : les phosphates mono- et bi-potassiques et, d'autre part, parmi les éléments colloïdes : le caséinate de calcium.

Voyons comment joue le caséinate de calcium dans la détermina. tion par titrimétrie de l'acidité du lait; nous n'indiquerons ici que le principe qui nous guide, les détails devant prendre place dans un travail qui paraîtra ultérieurement.

Le chiffre qui traduit le degré d'acidité titrable du lait dépend uniquement de l'indicateur coloré employé. Si on se servait d'hélianthine qui vire pour un $p \mathrm{H}=4,4$, le lait apparaîtrait comme alcalin. Avec la phénol-phtaléine, l'indicateur couramment employé qui commence à virer pour un $p \mathrm{H}=8,2-8,3$, le lait est de réaction acide. Le caséinate du lait ayant un $p \mathrm{H}=7$ ou aux environs de 7 , légèrement $>7$, il est clair, lorsque nous allons passer de 7 à $8,2-8,3$, que nous aurons besoin d'une certaine quantité de soude pour faire virer la phtaléine du phénol, et cette quantité exprimée en degrés Soxhlet ou Dornic traduit la part qui revient dans la titrimétrie du lait au caséinate lui-même.

Les méthodes qui ont pour but la détermination del'aciditédes caséines industrielles trouveront leur principe dans ce qui vient d'être dit.

Avec Mlle J. BRIgando (211), nous avons, en nous appuyant sur les données que nous venons d'exprimer à propos de la salification de la caséine par la soude ou la chaux, défini ce qu'il fallait exactement entendre par acidité de la caséine lactique et acidité de la caséine à la présure. Nous en exposons ici l'essentiel.

1 gramme de caséine pure, caséine obtenue bien entendu par précipitation acide, exige, avons-nous vu, pour déterminer le virage de la phénol-phtaléine $9 \mathrm{~cm}^{3}$ d'aleali $\mathrm{N} / 10$ environ. Nous donnons d'ailleurs le tableau qui résume sur ce point les résultats obtenus par plusieurs chercheurs.

\section{TABLEAU XXVIII.}

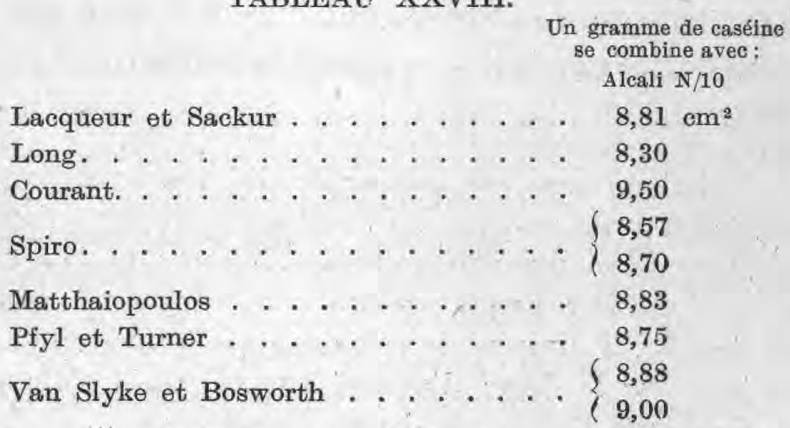

(211) Ch. Porcher et Mile J. Brigando. - Les bases scientifiques de l'analyse des caséines commerciales. Congrès de l'Assoc. fr. pour l'Avano. des Sciences, - Session de La Rochelle, 1928, et Le Lait, 1929, 9, 134. 
Si nous avons 30 grammes de caséine au litre, il faudra done $270 \mathrm{~cm}^{3}$ de soude $\mathrm{N} / 10$ ou $243 \mathrm{~cm}^{3}$ de soude Dornic $\left(4,5 \mathrm{grs}\right.$ de soude au litre). $10 \mathrm{~cm}^{3}$ de la solution de caséinate ainsi neutralisée à la phtaléine du phénol par la soude auraient exigé $2,43 \mathrm{~cm}^{3}$ de soude $\mathrm{N} / 10$; en langage industriel, on dira que la caséine au taux indiqué a $24^{\circ} 3$ d'acidité $\mathrm{D}$.

Si au lieu de prendre de la caséine pure, nous partons d'une caséine partiellement salifiée, notamment du caséinate calcique de $p H=7$, la quantité de soude qu'il faudra pour passer de ce $p \mathrm{H}=7$ au $p \mathrm{H}=8,2-8,3$ est évidemment moindre. Mais voyons d'abord ce qu'exige, traduit en degrés $\mathrm{D}$, avec l'emploi de la soude, la neutralisation partielle de la caséine par la chaux jusqu'à un $p H$ aux environs de 7 . Les documents de Patmer vont nous permettre de le dire.

30 grammes de caséine réclament en chiffres ronds $60 \mathrm{C}$ milligrammes de chaux pour donner un $p H=7$ ou aux environs immédiats de 7 . Titrimétriquement, ces 600 milligrammes de chaux correspondent à 857 milligrammes de soude. Tout à 1'heure, pour arriver au $p H=8,2-8,3$, il fallait $270 \mathrm{~cm}^{3}$ de soude $\mathrm{N} / 10$, soit pondéralement 1080 milligrammes. La différence, 223 milligrammes, marque la quantité de soude nécessaire pour que 30 grammes de caséinate de calcium de $p H=7$ qui est le caséinate normal du lait fassent virer la phénol-phtaléine. Or, ces 223 milligrammes se trouvent dans $50 \mathrm{~cm}^{3}$ de soude Dornic ; $10 \mathrm{~cm}^{3}$ de la solution du sus-dit caséinate auront donc vis-à-vis de la phtaléine du phénol une acidité de $5^{\circ}$ Dornic.

Bien entendu, nous sommes toujours dans l'hypothèse de caséine ou de caséinate très purs.

Si la caséine ou les caséinates renferment de l'eau, des impuretés salines, les ehiffres ci-dessus doivent être diminués dans une proportion dépendant du taux des impuretés et de l'humidité résiduelles.

Dans le dosage de l'acidité des caséines, la terminologie n'est plus la même que lorsqu'il s'agit du lait, puisque l'acidité donnée est exprimée en grammes d'acide lactique pour 100 grammes de caséine. Mais le raisonnement que nous venons de tenir nous permet de comprendre la différence qui existe entre l'acidité de la caséine-présure et celle de la caséine-acide. Nous y reviendrons d'ailleurs avec tous les détails voulus dans le travail que nous avons annoncé.

\section{L'ACTION DES SELS CALCIQUES SUR LES CASÉINATES}

Nous réservons pour un chapitre ultérieur, quand nous étudierons le mécanisme de l'emprésurage, l'action de la présure sur les caséinates de $p \mathrm{H}=7$ et $<7$ et les modifications chimiques et physiques que ce processus détermine; chimiques, quand nous envisageons la transformation du caséinate en paracaséinate avec.séparation d'un très petit morceau qu'il faudra mesurer; physiques, si nous nous retournons du côté de la viscosité par exemple. Evidemment, l'essentiel des phénomènes de l'emprésurage réside dans l'attaque du caséinate par le lab; nous devons done en reporter l'examen plus loin.

Il nous reste à étudier dans ce chapitre l'action de certains sels sur les caséinates. Dans la première partie de cette étude, nous avons 
rappelé quels étaient les sels précipitants les plus couramment employés pour séparer dans le lait les trois protéines essentielles : caséine, albumine et globuline ; nous ne reviendrons donc pas sur ce point. Rappelons que l'action de ces sels s'exerce complètement sur le caséinate qui est toujours précipité en entier. Présentement, nous nous limiterons à l'action des sels de calcium sur le caséinate calcique du lait.

ACTION DES SELS DE CALCIUM SUR LE CASÉINATE DU LAIT. Le caséinate ealcique est un électrolyte dont l'ionisation quoique faible est incontestable. D'autre part, quand deux sels, simultanément dissous dans un liquide, possèdent un ion commun, on sait que la présence de chacun d'eux diminue la solubilité de l'autre et son ionisation. Que l'un de ces sels ait l'aspect colloïdal, cela ne saurait modifier le sens du phénomène. Ces remarques, rappelées de la chimie générale, sont d'application au lait puisque dans ce liquide, à côté du complexe : caséinate de calcium + phosphates de calcium, déjà lui-même riche en chaux, se trouve à l'état cristalloïde du citrate de calcium. Il est certain que si l'on apporte au lait une charge supplémentaire calcique sous la forme soluble bien entendu, notamment celle de chlorure calcique, une tendance à la précipitation du caséinate va se dessiner.

Quand on s'adresse au lait, le phosphate insoluble de calcium, en même temps que le caséinate, précipitera; mais ceci n'est qu'un épiphénomène sans intérêt en ce moment pour nous.

Nous verrons tout à l'heure l'aide que l'élévation de la température apporte à la précipitation du caséinate de calcium

L'obtention du sérum chloruro-calcique. - Avec le lait, on élève quelquefois la température jusqu'à celle du B. M. bouillant, c'està-dire à un degré tel que l'albumine et la globuline sont précipitées presque totalement. Dans ces conditions, le bloc obtenu par addition du chlorure calcique est très complexe, puisqu'à côté du caséinate et des phosphates calciques, il renferme un coagulum albumineux. La liqueur résiduelle qu'on appelle le sérum chloruro-calcique servira à doser le lactose par réfractométrie (82).

La concentration des molécules dans le lait. - Pour comprendre la très grande sensibilité, accrue sous l'influence simultanée de la chaleur, du caséinate de calcium vis-à-vis de l'addition de sels calciques, notammentdu chlorure de calcium, sel le plus fréquemmentemployé, il est utile d'appeler l'attention sur une notion dont nous avons été le premier peut-être à relever l'importance ; c'est celle du degré de concentration des molécules qui se trouvent dans le lait, soit à l'état de solution vraie, soit à l'état de pseudo-solution.

Si l'on admet que la caséine ait un poids moléculaire minimum de 12.000 , nous voyons que sa concentration moléculaire dans le lait est extrêmement faible, puisque, pour un poids moyen de 30 grammes par 
litre, nous n'avons que $30 / 12.000$, soit $23 / 10.000$ de molécule. Si nous devions accepter des poids moléculaires plus élevés, - et nous aurions d'ailleurs raison, puisque, d'après CoHN, le chiffre de 12.000 est un chiffre minimum que nous avons choisi pour ne pas avoir à raisonner sur de trop grands nombres, - nous voyons que la concentration moléculaire de la caséine dans le lait serait encore beaucoup plus faible. Pour le poids formidable maximum de 192.000 auquel semble s'arrêter CoHN, nous n'aurions une concentration que de $15 / 100.000$. Ceci nous fait déjà comprendre que la dilution d'une solution de caséinate n'en peut augmenter que faiblement l'ionisation; puisque celle-ci dépend de la concentration, et que celle de la caséine dans le lait est vraiment faible, la dilution ne peut l'affecter que fort peu. Toutefois, dans le caséinate, il y a également de la chaux dont la concentration moléculaire n'est pas aussi faible que celle de la caséine; c'est pourquoi la dilution du caséinate favorise la libération de la chaux du sel calcique dont le $p \mathrm{H}$ marche ainsi, quoique faiblement, vers l'alcalinité.

Avec un caséinate à 600 , nous voyons qu'en face de 30 grammes de caséine, nous avons 600 milligrammes de chaux, soit en chiffres ronds, 1/100 de molécule, alors que tout à l'heure nous n'avions avec un poids molésulaire 12.000 que : 25/10.000 de molécule de caséine. Le rapport de ces concentrations est approximativement 4 . Il y aurait done 4 molécules de chaux en face de 1 molécule de caséine. Si nous prenions le poids moléculaire de 192.000 , nous aurions 66,6 molécules de chaux pour 1 molécule de caséine. Il y a loin du rapport 4 au rapport 66,6 , et si le premier est certainement trop faible, le deuxième est peut-être trop fort. Avec un poids moléculaire qui serait environ quatre fois le poids minimum 12.000 , le rapport ci-dessus deviendrait environ 16 .

Quoi qu'il en soit, ces chiffres sont extrêmement intéressants à confronter. Ils nous montrent que, dans le caséinate de calcium, nous avons toujours vis-à-vis d'une molécule de caséine, un nombre beaucoup plus grand de molécules de chaux, le nombre 4 étant certainement inférieur à la réalité, et le nombre 16 pouvant être choisi.

Pondéralement, nous avons dans le caséinate à 600,50 fois plus de caséine que de ehaux; mais les lois de la mécanique chimique jouent sur des concentration de molécules et ce sont ces dernières seules qui importent.

Par conséquent, quand on ajoute d̀ une solution de caséinate une quantité de $\mathrm{CaCl}^{2}$ qui pondéralement paraît assez faible, en fait, on modifie grandement la concentration de la chaux en présence. On l'augmente dans des proportions considérables, si bien qu'on réduit fortement l'ionisation du caséinate calcique et qu'on facilite son insolubilisation.

Un exemple emprunté à la technique de la réfractométrie du lait est très suggestif. Pour préparer le sérum du lait en vue de l'examen au réfractomètre, on peut précipiter la caséine, soit par l'acide acétique, 
soit par le chlorure de calcium. Arrêtons-nous à cette dernière technique qui des deux est la seule à illustrer les considérations dans lesquelles nous venons d'entrer.

A $30 \mathrm{~cm}^{3}$ de lait, on ajoute $0,25 \mathrm{~cm}^{3}$ d'une solution concentrée de $\mathrm{CaCl}^{2}$ contenant 200 grammes du sel fondu par litre, et l'on porte au B.M. bouillant pendant 15 minutes. On laisse refroidir et l'on filtre. Le filtrat est ensuite examiné au réfractomètre. Les calculs nous montrent que la quantité de $\mathrm{CaCl}^{2}$ qu'il faudrait ajouter à un litre de lait serait de $1 \mathrm{gr} .666$; calculée en chaux, cela ferait $0 \mathrm{gr} .840 .0$, dans le caséinate calcique du lait, nous n'en avons que 0 gr. 600. L'apport calcique est done plus que notable.

Nous allons revenir sur ce point un peu plus loin, mais retenons déjà que, tous calculs étant faits sur le nombre des molécules, nous pouvons dire que si tout à l'heure dans le caséinate de calcium, il y avait 16 molécules de chaux en face de 1 molécule de caséine, l'apport dans le lait de $\mathrm{CaCl}^{2}$, selon la quantité indiquée ci-dessus, augmente considérablement le nombre des molécules de la base; 22,4 molécules nouvelles de chaux sont ajoutées. C'est là une masse vraiment importante qui facilite la précipitation du caséinate calcique.

Toute une série de recherches que nous avons faites sur la façon dont le caséinate se comporte vis-à-vis du $\mathrm{CaCl}^{2}$ vont maintenant être exposées.

On sait que les caséinates sont stables à la température ordinaire, et même à l'ébullition, à la condition toutefois que leur alcalinité ne soit pas telle qu'il en résulte une décomposition de la caséine par l'excès de base, mais si l'on charge les solutions de caséinates de $\mathrm{CaCl}^{2}$, cette stabilité va disparaître. Le point où il y aura rupture d'équilibre, c'està-dire précipitation, va dépendre :

a) De la teneur initiale du caséinate en chaux;

b) De l'importance de l'apport en $\mathrm{CaCl}^{2}$;

c) De la température à laquelle on va porter le système.

Le premier facteur implique fatalement que l'influence du $p H$, qui dépend directement du taux de la base, doit être primordiale. Aussi, avons-nous opéré sur trois caséinates : à 400 , à 580 et à 800 , le premier, acide, le second neutre, le troisième alcalin, potentiométriquement parlant.

Quelques remarques préliminaires paraissent ici indiquées. Quand on ajoute du $\mathrm{CaCl}^{2}$ au lait, l'apport du sel introduit conduit nécessairement à des doubles décompositions avec les sels normaux préexistants, mais, objectivement, les modifications ne sont pas considérables, et ces doubles décompositions, à la température ordinaire, du moins pour des doses faibles de $\mathrm{CaCl}^{2}$, n'entraînent pas de précipitation.

Si nou's nous adressons au caséinate pur, on est débarrassé des sels parasites qui l'entourent dans le lait et, a priori, les résultats que nous relèverons pourront être plus facilement interprétés. 
LeS divers ChLoruRES de calcium. - En opérant sur le lait, nous nous sommes rendu compte combien il importait d'être fixé sur la qualité $d u \mathrm{CaCl}^{2}$ employé. Les solutions du $\mathrm{CaCl}^{2}$ ont des réactions tout à fait différentes, selon que le chlorure est cristallisé ou fondu. La solution de $\mathrm{CaCl}^{2}$ fondu est alcaline à la phtaléine du phénol, celle du $\mathrm{CaCl}^{2}$ cristallisé, acide.

Voici les $p H$ des solutions de ces deux sels à 50 grammes par litre :

$\begin{array}{ccc}\text { fondu } & \mathrm{CaCl}^{2} & \\ 9,20 & \text { cristallisé. } \\ & 5,55\end{array}$

Deux solutions de $\mathrm{CaCl}^{2}$ ont été préparées, l'une de sel fondu à 200 grammes par litre, et l'autre de cristallisé à 163 grammes par litre. Les quantités ajoutées au lait ont toujours été moléculairement les mêmes et il était tenu compte de la petite dilution qu'entraînait l'apport du $\mathrm{CaCl}^{2}$ au lait, dilution négligeáble lorsqu'il s'agissait de $\mathrm{CaCl}^{2}$ à un taux faible, moins négligeable lorsque l'apport chloruro-calcique était important.

TABLEAU XXIX.

$p$ H DU LAIT AdDITIONNE DE CaCle.

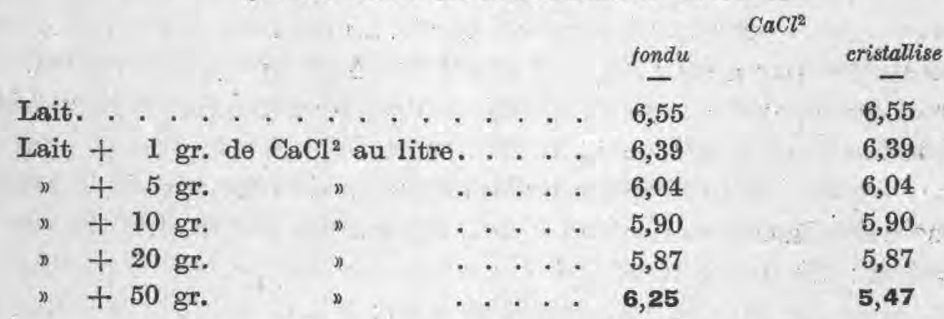

Nous voyons donc que pour les doses allant jusqu'à 20 grammes, la différence entre les deux $\mathrm{CaCl}^{2}$ ne fait pas sentir son action sur le $p \mathrm{H}$, mais dès que cette dose est atteinte et qu'on la dépasse, la différence est nette et le lait additionné de $\mathrm{CaCl}^{2}$ fondu retourne vers l'alcalinité.

La même expérience a été refaite sur un lait de $p H=6,90$.

Lorsqu'on ajoute du $\mathrm{CaCl}^{2}$ fondu et cristallisé à la dose de 50 grammes, à froid, les laits conservent leur aspect homogène, on ne note aucun précipité. A $40^{\circ}$ on a une fine floculation.

Pour d'autres laits, nous obtiendrons des précipitations pour des teneurs moindres en $\mathrm{CaCl}^{2}$; or, ces laits sont plus acides. L'acidité est un facteur qu'il y a lieu de faire intervenir.

Il est évident qu'il s'agit là de doses considérables dont l'intérêt n'est pas grand dans le genre de recherches que nous rappelons ici. Les doses de $\mathrm{CaCl}^{2}$ jusqu'à 5 gr. au maximum nous paraissent seules intéressantes. Au-dessus, les variations que nous signalons dans les phénomènes antérieurement observés sont beaucoup plus faibles. Il 
est dono vraisemblable que les observations relevées par L. PANCHAUd et certains autres auteurs sur le retour vers l'alcalinité des laits que l'on charge fortement de $\mathrm{CaCl}^{2}$ résultent de l'emploi qui a été fait de $\mathrm{CaCl}^{2}$ fondu.

Il était intéressant de préparer du chlorure de calcium tout à fait neutre. A. BARILLÉ (212) dans sa thèse sur les phosphates de calcium l'obtient de la façon suivante :

“ Les solutions de chlorure de calcium ayant généralement une réaction légèrement acide ou alcaline, nous avons préparé une solution neutre, titrée par ellemême, en dissolvant dans de l'acide chlorhydrique pur et dilué 5 grammes de carbonate de calcium précipité, chimiquement pur et bien sec. La solution a été évaporée à siccité pour chasser l'excès d'acide, et le résidu repris par une quantité suffisante d'eau distillée a été amené au volume d'un litre renfermant 5 gr. 55 de chlorure de calcium. Le titre de cette solution a été vérifié en dosant, ̀̀ l'état de sulfate calcique, la quantité de calcium contenue dans un volume de $50 \mathrm{~cm}^{3}$. On a obtenu 0 gr. 331 de sulfate calcique au lieu de 0 gr. 340, chiffre théorique n.

C'est, sauf indication contraire, le chlorure de calcium neutre préparé selon la méthode de Barillé qui a été utilisé dans la plupart de nos recherches.

Nous donnons ci-dessous les $p \mathrm{H}$ des solutions des trois chlorures, en solution concentrée et en solution diluée, au taux auquel ces chlorures sont employés par nous : 1 gr., 2 gr., 3 gr., 4 gr. et 5 gr. par litre.

L'eau qui a servi à faire les solutions des chlorures est de l'eau bidistillée bouillie dont le $p \mathrm{H}=5,33$.

Nous avons employé trois solutions mères de chlorure de calcium : $1^{\circ}$ La solution neutre de BARILLÉ à 111 grammes par litre $(\mathrm{N} / 1)$ dont le $p \mathrm{H}=6,53 ; 2^{\circ}$ La solution de chlorure cristallisé du commerce à 163 grammes par litre et dont le $p \mathrm{H}=5,99 ; 3^{\circ}$ Une solution de chlorure de calcium fondu à 200 grammes par litre qui est nettement alcaline à la phtaléine du phénol et dont le $p H=9,20$.

TABLEAU XXX.

$p$ HE SOLUTIONS DILUEES DES TROIS GHLORURES DE CALCIUM. Neutre (Barillé) Acide (Commerce) Alcalin (fondu)

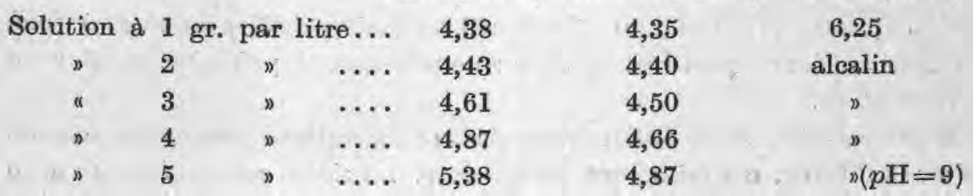

Nous constatons que le fait de dissoudre $\mathrm{du} \mathrm{CaCl}^{2}$ neutre ou acide, - le $\mathrm{CaCl}^{2}$ fondu, alcalin, ne pouvant être envisagé dans les présentes considérations, - suffit à abaisser le $p \mathrm{H}$ de l'eau. La dissociation du $\mathrm{CaCl} l^{2}$ fait donc virer ce dernier vers l'acidité.

(212) A. BARILLE. - Phosphates de calcium. Action de l'ammoniaque sur leurs dissolutions acides. Action de l'acide carbonique sous pression. - Thèse Doct. Univ. Paris, 1900. 
Cette remarque comporte des conséquences intéressantes. Nous verrons un peu plus loin, lorsqu'on ajoute du $\mathrm{CaCl}^{2}$ à un caséinate, que le $p \mathrm{H}$ de celui-ci marche vers l'acidité, et on doit alors se demander si cela ne doit pas être mis, soit en partie, soit en entier, sur le compte de la dilution pure et simple du $\mathrm{CaCl}^{2}$ ajouté.

Si nous nous adressons au chlorure de calcium fondu qui est alcalin, nous allons obtenir des chiffres intéressants qui nous laissent maintenant penser que le virage vers l'acidité pour les doses faibles résulte bien d'une réaction entre le caséinate de calcium et le $\mathrm{CaCl}^{2}$, et non pas de la dissolution pure et simple de ce sel fondu dans l'eau.

TABLEAU XXXI.

ADDITION DE $\mathrm{CaCl} l^{2}$ FONDU A UN CASÉINATE.

$\begin{array}{rlcc} & & \begin{array}{c}\text { Electrode à } \\ \text { hydrogène, }\end{array} & \begin{array}{c}p \text { H } \\ \text { Electrode à } \\ \text { quinhydrone. }\end{array} \\ \text { Caséinate de calcium. . . . . . . . . . } & 6,62 & 6,56 \\ \text { id. + } 1 \text { gr. } \mathrm{CaCl}^{2} \text { fondu (alcalin) . . . . } & 6,42 & 6,39 \\ \text { id. }+2 \text { grs. id. . . . . . . . . . } & 6,49 & 6,45\end{array}$

Comparativement, nous avons étudié ce qui se passe avec les solutions des mêmes quantités du même $\mathrm{CaCl}^{2}$ dans l'eau bidistillée et voici les chiffres obtenus :

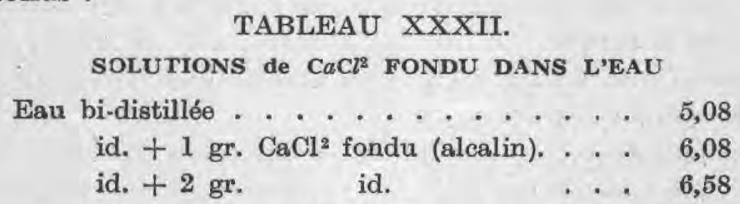

Nous voyons done que l'addition de $\mathrm{CaCl}^{2}$ alcalin qui fait virer nettement à la dose de 1 gramme par litre le $p H$ de l'eau bidistillée vers l'alcalinité, à la même dose, en présence du caséinate de calcium, ne fait virer le $p H$ que de peu, mais du moins vers l'acidité; dès qu'il $y$ a deux grammes de $\mathrm{CaCl}^{2}$, nous retournons vers l'alcalinité.

Comment expliquer ce virage vers l'acidité ? Si nous pouvions invoquer la formation d'un équilibre de Donnan entre les micelles de caséinate calcique et la phase aqueuse dans laquelle ils se trouvent plongés, cet équilibre exigerait qu'il y e ût plus de chaux dans le micelle que dans la phase aqueuse.

Lorsqu'on ajoute du $\mathrm{CaCl}^{2}$ à un caséinate, celui-ci blanchit et s'opacifie même. Comment interpréter ce blanchîment ? Sera-ce dans le sens d'une réaction chimique ? Selon les lois de GULDBERG et WAAGE, dès l'instant où l'on augmente la quantité présente de $\mathrm{Ca}$ soluble, on rend par cela même moins soluble le caséinate de calcium, on diminue son ionisation.

$\mathrm{Si}$ nous dialysons le système caséinate de calcium $+\mathrm{CaCl}^{2}$, nous enlevons le chlorure calcique additionné et nous revenons vers l'état 
initial, mais les phénomènes se compliquent ici de l'intervention d'un équilibre de DoNNan, dès l'instant où l'on opère par dialyse contre de l'eau fixe, - nous entendons par là, comme nous le verrons plus loin, la dialyse contre une quantité déterminée d'eau, et non pas contre de l'eau courante. Du chlorure de calcium passera à travers la membrane du dialyseur et un équilibre de Donnan va s'établir entre la solution qu'il donne à l'extérieur et la solution de l'intérieur faite du caséinate de calcium et du reste du $\mathrm{CaCl}^{2}$. Si on change l'eau, de nouveau du chlorure de calcium retenu du côté du caséinate dans la première dialyse va traverser la membrane et un nouvel équilibre de Donnan va s'établir. En continuant ainsi la dialyse, on retrouvera la totalité du chlore à l'extérieur. Les équilibres de DonNan qui pourraient jouer avec des dilntions extrêmement faibles de $\mathrm{CaCl}^{2}$ exigeraient des dosages de chlore et de chaux qui seraient en-dessous des erreurs expérimentales. Il semble donc que le rôle du chlorure de calcium lorsqu'on l'ajoute à une solution de caséinate de calcium est de déterminer la formation d'un nouvel équilibre, mais nullement permanent.

Revenons maintenant aux trois easéinates dont nous avons parlé plus haut : caséinates à 400,580 et 800 , et voyons comment ils se comportent après addition de chlorure de calcium.

Des parties égales des solutions des trois caséinates ci-dessus ont été additionnées de $\mathrm{CaCl}^{2}$ correspondant pour un litre de la liqueur à $1 / 2 \mathrm{~N} / 100,{ }_{4}^{3 / 4} \mathrm{~N} / 100,1,3$ et $5 \mathrm{~N} / 100$ de $\mathrm{CaCl}^{2}$; puis, on a porté à $40^{\circ}$ et à l'ébullition. Quelles sont les modifi. eations survenues dans les liqueurs ?

Si nous comparons les trois solutions, avant toute addition de $\mathrm{CaCl}^{2}$, nous constatons ce que nous savions déjà que le caséinate à 400 est plus blanc, plus opaque que celui à 580 , lequel à son tour est plus opaque que celui à 800 dont la translucidité, notamment contre une lumière vive, est très marquée.

Nous notons également que l'addition $d u \mathrm{CaCl}^{2}$ fait blanchir les caséinates; même avee une charge en ehlorure celcique de $1 / 2 \mathrm{~N} / 100$, la tendance à l'opacité est nette; elle s'accentue aveo l'importance de la charge en $\mathrm{CaCl}^{2}$. Voici d'ailleurs lo détail de l'expérience.

Caséinate à 400. - L'opacité de la liqueur additionnée de $5 \mathrm{~N} / 100$ de $\mathrm{CaCl}^{2}$ n'est pas plus grande que celle de la précédente qui ne contient seulement que $3 \mathrm{~N} / 100$ de $\mathrm{CaCl}^{2}$, mais on constate que l'opacité va en diminuant pour les trois premières solutions depuis celle qui ne contient que 1 N/100 jusqu'à celle qui ne renferme que $1 / 2 \mathrm{~N} / 100$.

Caséinates à $\mathbf{5 8 0}$ et $\mathbf{8 0 0}$. - Il n'y a pas de différences sensibles dans les cinq échantillons.

Avec le caséinate à 800 , la liqueur additionnée de $3 \mathrm{~N} / 100$ de $\mathrm{CaCl}^{2}$ précipite, slors que rien de semblable n'a pu étre noté pour les deux autres caséinates, à 400 et à 580 .

Opacité permanente des caséinates calciques additionnés d'un sel calcique soluble, puis chauffés. - Si l'on porte les tubes à $40^{\circ}$, on eonstate des faits intéressants. En dehors de certaines précipitations 
qui peuvent survenir, nous notons que dans toutes, les liqueurs deviennent beaucoup plus opaques qu'elles ne l'étaient déjà, plus laiteuses, et qu'elles ressemblent tout à fait à du lait écrémé également chauffé. Mais à l'inverse de ce que nous avons vu pour les seuls caséinates chauffés qui, refroidis, reprennent leur aspect premier, il n'en est plus ainsi après l'addition de $\mathrm{CaCl}^{2}$. Les caséinates qui sont devenus très opaques au B.M. à $40^{\circ}$, ne récupèrent plus leur apparence originelle lorsqu'on les refroidit. Il y a donc une modification permanente.

Caséinate à 400 (acide). - Les trois premières liqueurs additionnées respectivement de $1 / 2,3 / 4$ et $1 \mathrm{~N} / 100$ de $\mathrm{CaCl}^{2}$ restent homogènes, e'est tout au plus si l'on peut constater dans la dernière un précipité granuleux, presque insignifiant et dont on note la présence en inclinant avec soin le tube.

Avec $3 \mathrm{~N} / 100$ de $\mathrm{CaCl}^{2}$, on a une coagulation massive, comme un précipité d'emprésurage, mais mou et qui se disloque facilement par agitation.

Caséinate à 580 (neutre). - En portant au B.M. à $40^{\circ}$, on ne note, à part le blanchîment des liqueurs, rien de particulier ; aucun précipité, aucune coagulation. La liqueur additionnée de $5 \mathrm{~N} / 100$ de $\mathrm{CaCl}^{2}$ renferme un petit dépôt muqueux rassemblé au fond du tube, et il faut apporter le plus grand soin pour le constater.

Caséinate à 800 (alcalin). - Rien à signaler dans les trois liqueurs qui renferment respectivement : $1 / 2,3 / 4$ et $1 \mathrm{~N} / 100 \mathrm{de} \mathrm{CaCl}^{2}$ lorsqu'on porte à $40^{\circ}$; tout au plus, pour lo dernier, y a-t-il un petit dépôt granuleux au fond du tube.

Si nous allons à l'ébullition, voici que ce nous observons :

Caséinate à 400. - Les cinq tubes coagulent. Toutefois, dans les deux derniers, la coagulation est mieux marquée, le easéinate forme un chapeau à la surface, et le sérum est limpide, alors qu'il est encore louche pour les trois premiers.

Caséinate à 580. - Nous ferons les mêmes observations que pour le caséinate précédent, tout en notant cependant que le sérum est toujours louche.

Caséinate à 800. - Aucune différence dans les troís tubes, il n'y a pas de préeipitation, un léger dépôt muqueux sur le pourtour du tube, mais les liqueurs, très blanches, restent homogènes.

A $40^{\circ}$, le caséinate à 400 , acide, est moins stable que le caséinate à 580 qui est neutre, puisque avec $3 \mathrm{~N} / 100$, il précipite en formant un gel,alors que le caséinate à 580 ne précipite pas. Cette sensibilité plus grande du caséinate vis-à-vis du chlorure de calcium et que nous notons à $40^{\circ}$ s'exagère à l'ébullition, puisque le caséinate à 400 précipite mieux que celui à 580 .

Voici quels sont les $p H$ des trois caséinates sous l'action des apports variés de $\mathrm{CaCl}^{2}$ :

TABLEAU XXXIII.

$p$ DE GASEINATES DIFFÉRENTS PAR ADDITION DE DOSES DIFFÉRENTES DE $\mathrm{C} a \mathrm{C} l^{2}$.

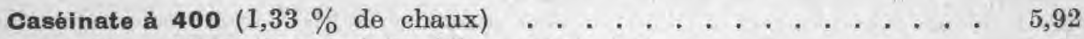

$+3 / 4$ moléeule $\mathrm{N} / 100 \mathrm{CaCl}^{3}(0$ gr. 83$) \ldots . . . \ldots . . . . .55,87$

1 (1 gr. 11) . . . . . . 5,84

$3 \quad$ $3 \quad(3$ gr. 33) ........... 5,71

$5 \quad$ (5 gr. 55) . . . . . . 5,61 


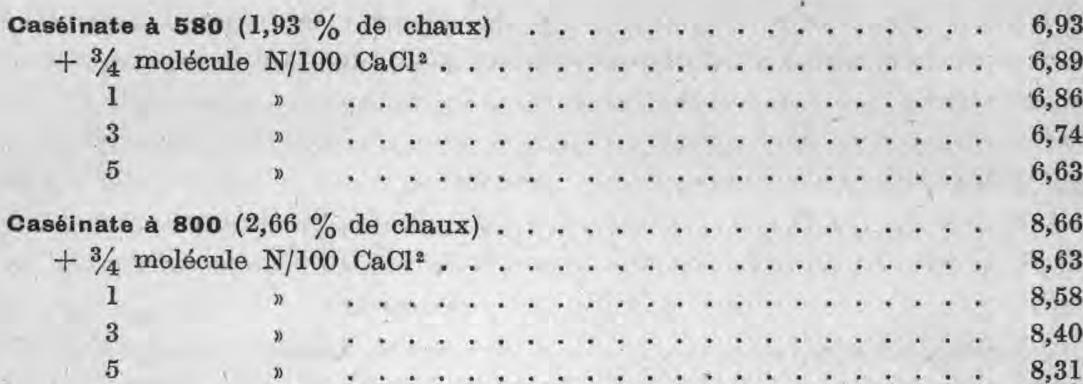

Avec ces trois caséinates, nous notons une marche vers l'acidité, mais elle est faible pour les premières doses. Avec l'addition de $1 / 2$ $\mathrm{N} / 100$, et nous avons même essayé $1 / 3 \mathrm{~N} / 100$ de $\mathrm{CaCl}^{2}$, nous n'avons pas noté de différence sensible avec le $p \mathrm{H}$ initial.

Ce que nous disons du $\mathrm{CaCl}^{2}$ dans les pages qui précèdent, nous pourrions le répéter de tout autre sel soluble de calcium, minéral ou organique: azotate, acétate, lactate, etc... car ici l'anion du sel importe peu, c'est le cathion seul qui joue.

Nous verrons plus loin, quand nous aurons en présence une masse suffisante de phosphate acide soluble de calcium ou de bicarbonate calcique, que ces sels, tout comme le chlorure, l'acétate, etc... cités plus haut, précipiteront le caséinate calcique lorsque les conditions favorables de concentration et de température seront atteintes; nous obtiendrons un coagulum ressemblant, encore une fois, au coagulum d'emprésurage, un peu plus mou peut-être, mais objectivement, les deux caillés seront comparables. N'est-ce pas ce que nous avons obtenu, à $40^{\circ}$, déjà avec le caséinate à 400 additionné de $3 \mathrm{~N} / 100$ de $\mathrm{CaCl}^{2}$ au litre?

LA PRÉCIPITATION DU LAIT PAR LE CHLORURE DE ZINC. Nous avons vu plus haut comment on obtenait le sérum chlorurocalcique en vue de son examen au réfractomètre. Plusieurs autres sels ont été utilisés pour précipiter, non seulement la caséine, mais l'albumine du lait en vue de la préparation de liqueurs limpides destinées également à la détermination de l'indice de réfraction. Dans sa thèse, A. LATAIX (82) les examine très judicieusement tour à tour.

Le chlorure de zinc méritait d'être étudié à ce point de vue.

Nous avons trouvé que ce sel précipite même à froid la caséine du lait à des doses plus faibles que celles qui sont nécessaires au chlorure de calcium pour obtenir le même résultat.

Nous avons cherché quelle était la quantité minimum de chlorure de zine, en 1/10 de milligramme nécessaire pour amener la précipitation de la caséine de $25 \mathrm{~cm}^{3}$ de lait à des températures différentes, en donnant un sérum clair. Le lait originel avait une acidité de $17^{\circ} \mathrm{D}$; le chlorure de zinc a été ajouté jusqu'à 
ce qu'on obtienne des grumeaux très nets, laissant entre eux un sérum limpide: on filtre.

Le graphique donne ces quantités. Nous voyons qu'il faut, à la température de $40^{\circ}$, tous calculs faits, $2,25 \mathrm{gr}$. de chlorure de zine par litre de lait pour amener la précipitation de la caséine avec obtention d'un sérum propre à la détermination de l'indice de réfraction.

\section{MOYENS D'ARRIVER AU SYSTEME : CASÉINATE DE CALCIUM + CHLORURE DE CALCIUM}

Il est deux manières d'arriver au système : caséinate de calcium + $\mathrm{CaCl}^{2}$ :

Caséinate calcique acide $+\mathbf{C a C l}^{2}$. - La première, dont nous avons implicitement parlé dans les pages qui précèdent est de prendre un caséinate d'une charge en chaux donnée, mais d'un $p H \leqslant 7$, et d'y apporter un quantum déterminé de $\mathrm{CaCl}^{2}$ calculé anhydre.

Caséinate calcique alcalin + $\mathbf{H C l}$ - La seconde, est de prendre un caséinate calcique alcalin, puis de neutraliser l'excès de chaux par $\mathrm{HCl}$.

Il est évident que si les deux systèmes, une fois préparés, ont en présence les mêmes masses de caséine, de chaux et de chlore, nous aboutissons à un même édifice dans un cas comme dans l'autre. Quel que soit le modus faciendi adopté, nous devons arriver au même $p H$ et à une réaction identique vis-à-vis de la présure dans les mêmes circonstances. C'est ce que nous verrons dans la suite de ce travail.

Qu'arrivera-t-il, en partant d'un caséinate calcique de réaction très alcaline, d'un $p H$ nettement $>7$, si, au lieu de neutraliser l'excès de chaux par $\mathrm{HCl}$, c'est-à-dire un acide conduisant à un sel calcique qui est soluble, nous employons des acides polybasiques : $\mathrm{PO}^{4} \mathrm{H}^{3}, \mathrm{CO}^{4} \mathrm{H}^{2}$ pouvant conduire à l'obtention de sels, les uns, solubles : phosphate mono-calcique et bicarbonate calcique qui sont très solubles, phosphate bi-calcique, moins soluble ; les autres, insolubles : phosphate tricalcique et carbsnate calcique neutre? Nous aboutirons à des systèmes plus compliqués que le système : easéinate de calcium + chlorure de calcium et dans lesquels, contre le caséinate calcique, nous aurons à côté les uns des autres, des aspects, les uns solubles, les autres insolubles, des sels calciques néoformés et dont les proportions relatives dépen. dront des masses en présence et de la température.

Pour y voir clair, notamment lorsqu'il s'agira de neutraliser l'excès de chaux du caséinate calcique très alcalin par l'acide phosphorique, il importera de reprendre l'étude de la mécanique des phosphates calciques, aux doses que nous avons à manier, en étudiant l'allure des réactions des liqueurs par la détermination de leur $p \mathrm{H}$. 


\section{COURBE I}

QUANTITÉ DE Z $n$ C $l^{2}$ EN FONGTION DE LA TEMPÉRATURE POUR L'OBTENTION D'UN SÉRUM CLAIR AVEC DU LAIT.

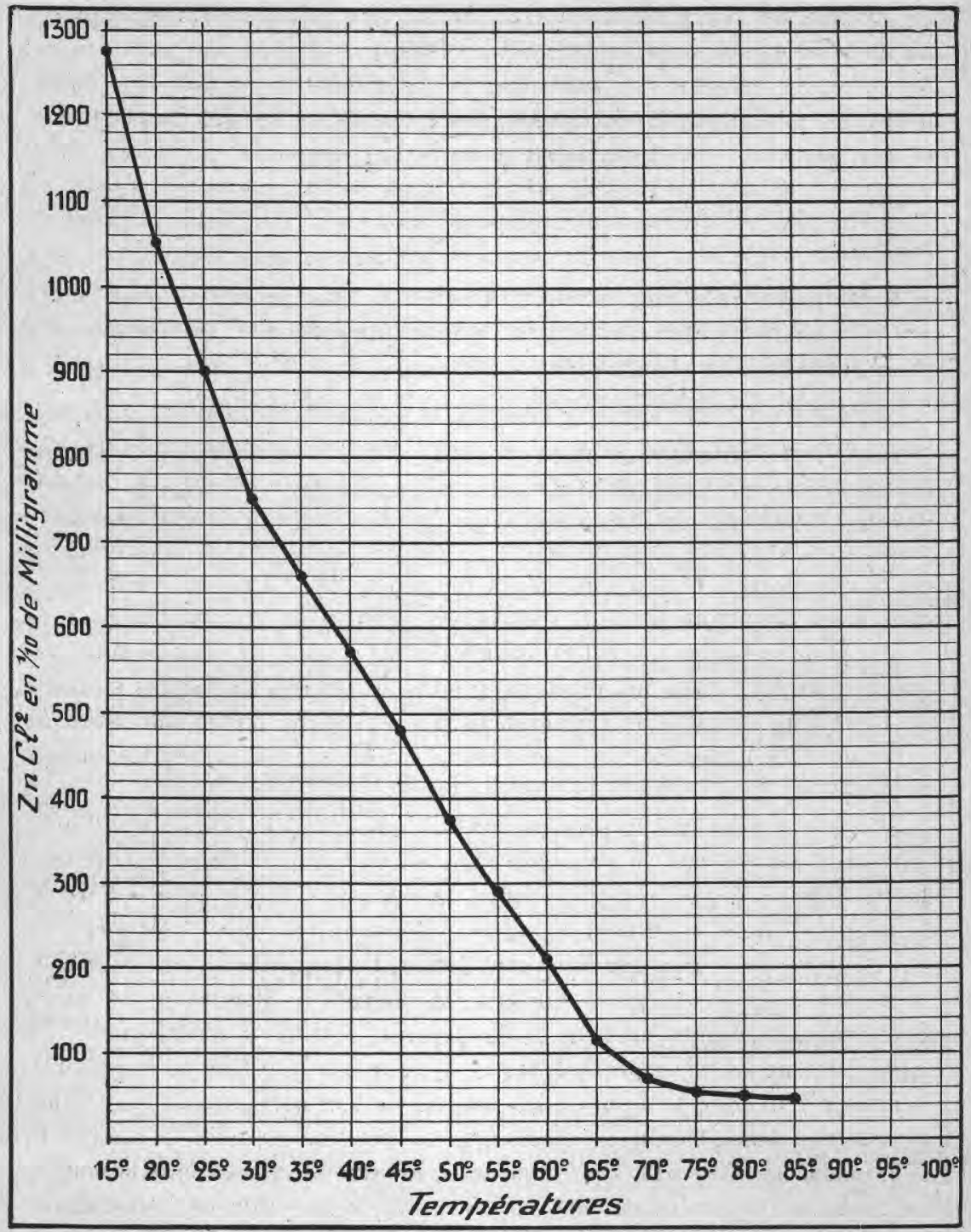




\section{CHAPITRE VI.}

\section{LA PRÉSURE}

Depuis un temps immémorial, on sait qu'une macération aqueuse de la caillette du veau, - ainsi d'ailleurs que de tous les estomacs des mammifères allaités, - provoque la coagulation du lait légèrement tiédi. La fabrication des fromages, vieille comme le monde, peut-on dire, repose sur cette réaction. C'est avec le lait de vache, de brebis et de chìre, - et que la présure soit d'origine bovine, ovine ou caprine, il importe peu, - que le phénomène se présente à nous sous la forme la plus nette. On a beaucoup discuté sur son essence, mais nous reporterons plus loin l'examen des hypothèses qui ont été soulevées à son sujet. Un seul point ici sera retenu par nous : c'est celui de l'identité ou non de la présure et de la pepsine.

PRÉSURE ET PEPSINE. - La caillette est, anatomiquementet physiologiquement, l'estomac du veau; or, le propre de l'estomac est de sécréter d'une part, l'acide chlorhydrique, d'autre part, un ferment : la pepsine lequel procède en milieu acide à la première attaque des molécules protéiques ingérées. La digestion pepsique simplifie la structure de ces dernières qu'elle disloque en polypeptides plus ou moins compliqués, sans aller toutefois jusqu'à mettre en liberté des acides-aminés; du moins pour le temps qui est consacré à cette digestion, il ne semble pas que l'on trouve dans les produits rendus libres des molécules simples. Ce n'est que in vitro, en laissant la pepsine faire porter son attaque pendant de longues heures que l'on peut relever, et encore en faibles proportions, la libération d'acides-aminés.

C'est au x ferments du pancréas et de l'intestin, à la trypsine, à l'érepsine, qu'il est réservé de procéder à un broyage moléculaire plus poussé des molécules, lequel conduit alors à la mise en liberté, et rapidement, d'un grand nombre d'acides-aminés. Reconnaître dans l'estomac du veau, à côté de la pepsine, un autre ferment : la présure, dont le rôle se limiterait à la coagulation du lait, la digestion ultérieure du caillé formé ressortissant à l'action de la pepsine, semble a priori facile. La pspsine est un ferment protéolytique, - il en est de même de la présure, - mais si l'on cherche à suivre les progrès de leur attaque sur la caséine, on observe des différences. Alors que la pepsine liquéfie le coagulum d'emprésurage, il semble que la présure, lorsqu'elle a provoqué la coagulation du lait, s'arrête là. Son inertie vis-à-vis du caillé est indiscutable, du moins dans les conditions où l'on opère habituellement. En d'autres termes, l'action de la pepsine est plus poussée que celle de la présure. Alors que la présure se contente de détacher de la molécule protéique un faible morceau que nous verrons représenter $4-5 \%$ toutau plus de 
celle-ci, la pepsine, au contraire, procède à une dislocation plus complète.

La rapidité d'action de la présure. - La pepsine et la présure ne s'opposent pas seulement l'une à l'autre par la nature de leur action dissociante sur la caséine, mais également par la rapidité de cette action. Dès l'instant que la solution du ferment-lab est remarquablement active, en d'autres termes, très concentrée, il suffit de quelques secondes pour provoquer la eoagulation du lait par la présure. C'est même cette rapidité qui avait fait mettre en douto que la présure pît être un ferment. On oubliait qu'il est possible de préparer des amylases d'une formidable activité, ne mettant également que quelques secondes pour détacher des moléoules de sucre de la matière amylacée préalablement cuite pour la rendre plus sensible à l'attaque de la diastase hydrolytique. Avec la pepsine, la digestion du caillé demandera plusieurs quarts d'heure, plusieurs heures même.

Lorsqu'on regarde au fond des choses, on voit que la distinction que nous voulons faire entre la présure et la pepsine n'est pas d'une très grande solidité. Quand nous parlons de la présure, nous notons que très vite se sépare de la easéine un petit morceau représentant, avons. nous dit, 4-5 \% de l'ensemble. Quand nous parlons de la pepsine, nous avons tendance à ne juger son action digérante que lorsque nous voyons les caillots préformés se dissoudre graduellement. En fait, avant que nous n'arrivions à cette digestion visible, véritable liquéfaction, il est certaîn que la pepsine a déjà attaqué la caséine, peut-être même en plusieurs points simultanément. Rien ne nous dit que l'attaque n'ait point été rapide; une échelle de mesures nous fait défaut. Quand nous voyons le caillé du lait se ramollir, nous sommes, après tout, presque vers la fin d'un processus.

Ce que nous voulons relever ici, c'est que dans les mêmes conditions de température et de temps, alors que la pepsine aura liquéfié entièrement le caillot de lait, la présure aura, depuis longtemps, cessé d'agir. Encore une fois, elle reste inerte en présence du produit de son action. Il semblerait donc que si l'action de la présure est uniquement coagulante, celle de la pepsine n'est que liquéfiante. Au fond, nous jouons sur les mots, parce que présure et pepsine sont toutes deux des ferments protéo. lytiques; mais alors que la protéolyse s'arrête très vite avec la présure, elle se poursuit au contraire avec la pepsine, et quand nous aurons disséqué l'action de la présure sur le lait, ce à quoi nous ne pourrons atteindre avec quelque exactitude qu'en opérant sur des édifices synthé. tiquement préparés, nous nous rendrons aisément compte que le mot coagulant s'il paraît être celui que l'ón doit choisir, en ne se basant que sur l'aspect grossier du phénomène, pourrait très bien être laissé de côté. La coagulation, c'est-à-dire le dépot de flocons qui se soudent, n'est que le second temps de la réaction; il en est le moins important. 
Or, on ne saurait dénier d'un autre côté à la pepsine une action dite coagulante du même ordre. Pendant la guerre, devant les difficultès que l'industrie des fromages éprouvait à se procurer des présures commerciales, beaucoup de pays ont été obligés de recourir à des macérations d'estomacs autres que les caillettes de veau. On a utilisé des macérations d'estomacs de porcs adultes prêts pour la boucherie et l'on a constaté qu'elles donnaient d'aussi bons résultats pour l'emprésurage du lait que les présures commerciales. Tout au plus, fallait-il prendre certaines présautions tenant à l'acidité du lait, à la dilution de la macération d'estomaes de pores et à la température de l'emprésurage. La pepsine des dites macérations jouạit le même rôle que la présure et rendait les mêmes services dans l'industrie fromagère.

L'identité d'origine de la présure et de la pepsine, l'analogie de leur fonction, puisque toutes deux sont des ferments protéolytiques, rendent a priori leur séparation difficile, si difficile même que la question de-l'identité de ces deux ferments peut être acceptée. A cette identité s'opposent toutefois les dissemblances d'action que nous avons relevées plus haut. Nous sommes donc amené à voir si la pepsine et la présure agissent vraiment dans les mêmes circonstances.

Nous n'avons tenu compte tout à l'heure, dans la comparaison que nous avons faite des actions de la présure et de la pepsine, que des circonstances de température et de temps, e'est-à-dire que faisant agir ces deux ferments à la même température, nous comparions la rapidité de leur action, indépendamment des modalités d'aspect de celle-ci. Or, nous avions provisoirement laissé de côté un facteur extrêmement important : la réaction du milieu.

LA RÉACTION DU MILIEU, - La présure agit aux environs de la neutralité; nous verrons même qu'avec un $p H=7,4-7,5$, - c'est là l'extrême limite, - elle coagule encore le lait.

La pepsine, au contraire, exige une forte acidité. Le suc gastrique des animaux renferme $2,3,4$ grammes et quelquefois plus de $\mathrm{HCl}$ par litre, ce qui répond à un $p \mathrm{H}$ très bas $(1,8$ à 2$)$.

Ce sue coagule le lait, ce qui ne doit pas nous surprendre, puisque deux facteurs unissent ici leurs actions pour faciliter cette coagulation le ferment et l'acidité, mais s'il est neutralisé, il ne perd pas son pouvoir coagulant. Par la neutralisation, on a éliminé le facteur acide, mais on a conservé le facteur pepsine ou le facteur présure.

Nous nous trouvons donc amené à nous demander si nous avons là deux ferments différents ou s'il ne s'agit que d'un même ferment dont l'action protéolytique, légère avec l'un, profonde avec l'autre, est conditionnée simplement par la réaction du milieu.

En milieu neutre, avec un $p H$ aux environs de 7 ou dépassant légèrement 7, nous n'aurions qu'un pouvoir protéolytique faible, très spécifique- 
ment orienté, se traduisant par la séparation d'un morceau représentant 4-5\% de l'ensemble. Dans les conditions ordinaires de la pratique, à ceci répondrait ce qu'on appelle la coagulation du lait, coagulation qui s'arrêterait là.

Au contraire, avec une acidité forte, un $p \mathrm{H}$ aux environs de 2 , nous voyons le ferment attaquer plus profondément la molécule protéique, en séparer des peptides plus ou moins gros, phosphorés, - du moins les plus gros, - puis les décomposer pour en faire de plus petits, sans aller toutefois jusqu'au terme acides-aminés. Le coagulum ne persiste pas, il s'est liquéfié.

Plusieurs auteurs ont fait remarqueer que le lait coagulé par la présure était plus facilement attaqué par la pepsine, mais après tout s'agit-il de deux actions successives relevant l'une et l'autre de deux ferments différents, ou bien n'avons-nous qu'un seul ferment commençant par coaguler, - voilà pour le côté présure -, puis poussant plus loin son action et digérant le caillé, - voilà pour le côté pepsine.

Nous verrons plus loin que si la présure agit en milieu neutre, sonaction est singulièrement facilitée par l'acidité. Il est vrai que dans les recherches qui soulignent l'influence favorisante de celle-ci, on n'a jamais mis en ceuvre que des acidités faibles, descendant rarement pour le lait audessous de $p H=6$. Mais il eût été intéressant d'opérer avec des acidités beaucoup plus fortes comme celles du suc gastrique; or, avec elles, on a toujours une précipitation purement acide de la caséine.

Quand nous venons de dire que tout dépend et uniquement de la concentration en ions $H$, qu'en somme pepsine et présure sont deux aspects du même ferment dont le mode d'action est conditionné justement par cette concentration, nous trouvons un exemple analogue chez une autre diastase hydrolytique qui s'attaque aux glucides. Bierry a montré que le suc pancréatique neutralisé, puis chauffé à $40^{\circ}$ pendant 30 minutes ne saccharifie plus l'empois d'amidon, mais le liquéfie encore, comme si son amylase, fait-il remarquer, se composait d'une diastase liquéfiante, mais non saccharifiante, et d'une amylase proprement dite, saccharifiante. L'amylase pancréatique dont l'action saccharifiante est maxima en milieu légèrement acide, cesse de mettre du maltose en liberté et ne donne que de la dextrine lorsqu'on opère en milieu neutre.

Il est vrai qu'on a toujours la ressource de dire, qu'il s'agisse de sue gastrique ou de suc pancréatique, qu'en réalité il y a bien deux ferments agissant chacun aux environs d'un $p H$ déterminé, et non pas un seul ferment qui, selon le taux du $p H$, agit tantôt d'une façon, tantôt de l'autre.

Deux Ecoles sont donc possibles et elles ont chacune leur chef : l'Ecole de l'identité ảe la présure ei de la pepsine avec PAwLow et ses 
élèves; l'Ecole de Hammarsten et de Bang qui estiment que pepsine et présure sont deux agents différents. (213, 214, 215).

Nous ne saurions dans ce travail nous étendre plus longuement sur cette question. Ce n'est pas que nous pensions qu'elle soit oiseuse, mais nous devons reeonnaître qu'il est très difficile d'opter nettement pour l'une ou l'autre de ces hypothèses en ayant la certitude de ne pas laisser derrière soi des arguments de valeur qui plaideraient en faveur de celle que l'on n'aurait pas acceptée.

La grande dispersion de la présure. - La présure est un ferment qui est très répandu, non seulement dans le règne animal, mais dans le règne végétal.

M. JAVILLIER (216) dans sa thèse a montré l'extrême diffusion de la présure chez les végétaux. Il a été suivi dans cette voie par Seldier (217), Gerber (218), Briot (219), etc...

Beaucoup de microbes sont également sécréteurs de présure (220).

C'est done reconnaître que ce ferment protéolytique est, d'une façon très générale, sécrété par la cellule vivante, que celle-ci appartienne au règne animal, au règne végétal ou aux infiniment petits. Nous ne voulons pas dire que e'est un ferment banal, mais son aire de dispersion est considérable. En signalant le fait, les théories finalistes confirmeraient par cela même son utilité, son indispensabilité, irions-nous jusqu'à dire; c'est peut-être aller un peu loin. En ce qui concerne les mammifères, ce serait admettre qu'il est absolument nécessaire que le lait soit toujours coagulé par la présure avant d'être liquéfié sous l'action des autres diastases digestives qui poussent beaucoup plus loin la protéolyse de la caséine. Or, la prise en masse du lait dans l'estomac du jeune est

(213) Sellier. - Identité du ferment protéolytique et de la présure. C. R. Soc. biol. $1908,2754$.

(214) J. Hawessox. - L'influence du lab ferment sur la maturation du fromage. Expériences avee le Backstein russe. - Le Lait, 1929, 9, 2. Dans ce travail, l'auteur fait un historique critique intéressant de la question soulevée ieí : l'identité ou non de la présure et de la pepsine.

(215) S. A. Waksman et W. C. Davison, - Enzymes, Properties, Distribution, Methods and Applications. The Williams and Wilkins Co, Ed., Baltimore 1926. Pour la présure, p. 197.

(216) M. Javnliner. - Oontribution à l'étude de la présure chez les véyétaux. - Thèse de Pharmacie. Paris 1904.

(217) J. Sfrluner. - Existence de la présure chez les invertébrés. (A phrodite aculeata). C. Ii. Soc. Biol., 1907 (1), 62, 693.

(218) Gerber. - Nombreuses eommunications sur les présures végétales, les présures des animaux inférieurs, les présures des mammifères, les conditions de leur action dans les $C$. R. Soc. Biol. pendant les années 1907-1910.

(219) A. Briot. - Sur la présure du figuier (Ficus carica). C. R. Soc. Biol. 1907 (1) 62, 972. Du même auteur, plusieurs communications dans le mème recueil sur la présure, la pepsine, la parachymosine, en 1907-1908.

(220) E. Duchaux. - Traité de Microbiologie : Diastases, Toxines et Venins. G. Masson et Cie, Ed. Paris. (Présure: p. 590.) 
loin de toujours affecter l'aspect que nous reconnaissons au coagulum du lait de vache emprésuré in-vitro. Des modalités très différentes de floculation, de consistance sont à relever, non seulement chez les diverses espèces, mais chez la même espèce. Cela tient-il uniquement au ferment ? Non, car la constitution colloïdale du lait, la structure de son ensemble salin, interviennent aussi pour modifier l'aspect du coagulum. C'est ce que nous verrons en nous adressant d'abord au lait, ensuite aux édifices synthétiquement préparés. Cela nous permettra d'attribuer à chaque facteur qui va jouer un rôle dans la coagulation du lait par la présure, l'importance qui lui revient.

Nous avons dit plus haut qu'avant d'entreprendre des recherches effectuées par voie synthétique, nous avions opéré sur le lait écrémé. Celles dont il va maintenant être fait état, datent de l'été 1923, il y a bientôt 6 ans. Ce sont des recherches préliminaires; elles nous étaient nécessaires pour nous entraîner, en quelque sorte, à l'étude de cette question et nous permettre de vérifier des données antérieurement acquises par d'autres en les reprenant sous un angle un peu différent parfois. Nous en parlerons uniquement comme des faits, nous gardant de la moindre interprétation des résultats. Ensuite, lorsqu'il nous sera possible de rapprocher ces recherches faites sur le lait de celles que nous aurons entreprises sur des édifices synthétiquement préparés, nous aurons à faire intervenir certaines hypothèses dans l'explication des phénomènes observés, ici et là.

Auparavant, nous croyons bon de parler de la dialyse.

\section{LA DIALYSE}

La dialyse est un processus physique très précieux à utiliser dans des recherches du genre de celles qui sont relatées ici. Que nous le fassions jouer sur la présure, le lait ou les complexes : caséinate calcique + un sel calcique, soluble ou non, il importe de prendre des précautions que nous allons maintenant mentionner.

La dialyse étant un processus lent, qui demande par conséquent beaucoup de temps, il est indiqué d'utiliser des antiseptiques, puisque nous avons affaire à des liquides facilement altérables par les microbes. Parmi eux, se présentent à l'esprit d'abord le formol, puis l'essence de moutarde, le thymol et le toluène.

Beaucoup des auteurs qui ont eu à effectuer des recherches sur la dialyse du lait se sont adressés au formol. Nous verrons que le formol ne gêne nullement l'action de la présure aux doses auxquelles il est indiqué de l'ajouter au lait afin d'en éviter l'altération pour permettre à la dialyse de produire tous ses effets.

Par la dialyse, on élimine des liqueurs les éléments minéraux solubles, on modifie par conséquent l'aspect et la masse de l'édifice salin résiduel dans un sens bien déterminé. Il sera possible, en effet, a près dialyse, 
d'analyser tantôt la partie qui a passé à travers la membrane du dialyseur, tantôt, au contraire, ce qui reste dans le dialyseur, et de le comparer à la liqueur originelle, qu'il s'agisse, encore une fois, de lait ou de milieux synthétiquement obtenus.

Dans le but d'être fixé sur les conditions dans lesquelles nous devons effectuer la dialyse pour en obten les meilleurs résultats, quatre séries de recherches ont été entreprises, portant respectivement sur :

1) La nature des antiseptiques auxquels on a recours;

2) La nature de la membrane du dialyseur;

3) La température à laquelle doit se faire la dialyse;

4) Le mode de dialyse, et ici nous entendons distinguer la dialyse vis-à-vis d'eau courante ou au contraire vis-à-vis d'un volume d'eau déterminé que, par opposition, nous appellerons eau fixe.

10 Les ANTiseptiques. - Le formol est un antiseptique qui tue très rapidement les ferments lactiques, $s$ 'attaquant moins aisément aux microbes sporulés qui sont plus résistants. C'est un fait connu que les laits formolés ne s'acidifient pour ainsi dire jamais, mais par contre se putréfient aisément.

L'inconvénient principal du formol est de s'unir à la caséine, mais il est à négliger dès l'instant où les effets de la dialyse sont envisagés surtout du côté salin.

L'essence de moutarde a l'avantage d'être un antiseptique très énergique en même temps que volatil. Elle n'a aucune action nuisible sur les constituants du lait, quels qu'ils soient. Nous l'employons d'une façon régulière dans nos recherches portant sur les édifices à base de caséine synthétiquement préparés, préférant recourir au formol lorsqu'il s'agit de lait, à la dose de 1 goutte de formol pur pour $100 \mathrm{~cm}^{3}$ de lait, dose qui doit être portée à II à III gouttes pour les laits non récoltés au laboratoire. On peut ainsi s'assurer la possibilité d'une dialyse pouvant durer plus de 24 heures, sans altération notable des constituants protéiques du lait.

Si nous employons l'essence de moutarde, nous prendrons également I ou II gouttes pour $100 \mathrm{~cm}^{3}$ de complexe caséinate de calcium + phosphate de calcium ou tout autre édifice salin dans lequel entre le caséinate de calcium.

S'il s'agit d'utiliser l'essence de moutarde pour le lait, nous devrons porter à III gouttes ou même IV gouttes la quantité de celle-ci.

Le thymol nous a donné aussi de bons résultats, surtout pour les complexes ou la présure ; un petit cristal déposé à l'intérieur du dialyseur assure une action antiseptique suffisante. On peut parfois associer l'action du thymol à celle de l'essence de moutarde.

Le toluène peut également être employé.

Malgré l'emploi des antiseptiques, il faut bien reconnaître, à moins de conditions toutes spéciales sur lesquelles nous allons revenir tout 
à l'heure, qu'il ne faut pas que la dialyse dure plus de 24 à 36 heures, du moins lorsqu'il s'agit de lait.

Avec cette sécrétion infailliblement polluée lors de la traite, la valeur d'un antiseptique, si grande soit-elle, ne peut être absolue. Les microbes les plus sensibles sont évidemment atteints par lui, mais restent malheu. reusement les microbes à spores, thermophiles, résistants et particulièrement protéolytiques. Ce sont eux qui interviendraient à la longue et qui altèreraient la liqueur si la dialyse devait se prolonger longtemps, d'autant plus, il ne faut pas l'oublier, que l'antiseptique dialyse aussi pour son propre compte, moins peut-être le formol, qui par ses aptitudes chimiques se fixe mieux sur les matières protéolytiques, et le thymol peu soluble que l'essence de moutarde volatile. Aussi, si l'on tient à ce que la dialyse se continue, il est indiqué de temps à autre, toutes les 12 heures, d'ajouter un peu de l'antiseptique choisi quelqu'il soit. Il est certain que lorsqu'on fait porter ses recherches sur des complexes obtenus au laboratoire, des précautions d'asepsie peuvent être prises qui permettent peut-être d'éviter l'emploi des antiseptiques. En effet, lorsqu'on prépare le complexe caséinate de calcium + phosphates de calcium, tous les réactifs que l'on met en présence : caséine, eau de chaux, acide phosphorique, sont stériles. La question ne se pose évidemment pas pour la chaux et l'acide phosphorique, mais la caséine, lorsqu'elle a été préparée par lavages successifs avec l'alcool, l'acétone et l'éther est devenue également un produit stérile. Si donc les flacons dans lesquels les réactions vont se passer pour aboutir au complexe sont stérilisés, on met de son côté toutes les chances pour que le complexe obtenu soit aussi peu pollué que possible, et dans ces conditions, il faudra infiniment peu d'antiseptique pour assurer l'asepsie de la dialyse.

$2^{\circ}$ LA MEMBRANE du dialyseur. - Dans nos toutes premières recherches, nous employions les dialyseurs SCHLEICHER et ScHÜLL, mais devant l'irrégularité de leur pâte, de la lenteur apportée à la dialyse tenant à leur forte épaisseur, nous leur avons substitué les dialyseurs au eollodion fabriqués au laboratoire selon une technique bien connue.

Avec eux, on peut réaliser toutes les perméabilités voulues; il suffit pour cela de faire varier le nombre des couches et, aussi, d'employer des solutions de collodion plus ou moins concentrées, ce qui permet d'agir sur les dimensions de leurs pores.

Malgré le soin apporté à la préparation des filtres, il est difficile, toutes conditions égales d'ailleurs, de garantir une égalisation parfaite de leurs qualités. C'est là une observation d'ordre général bien connue et souvent exprimée qui n'empêche nullement de tirer des filtres en collodion les grands avantages qu'on leur reconnait dans les recherches sur la dial yse. Leur uniformité apparente est du moins facilement obtenue et la dialyse est rapide. 
La capacité des dialyseurs peut varier considérablement, de $10 \mathrm{~cm}^{3}$ jusqu'à 100 à $150 \mathrm{~cm}^{3}$; nous en avons même préparé qui pouvaient contenir $250 \mathrm{~cm}^{3}$ de liqueur; tout cela dépend de la grosseur du mandrin que l'on emploie. Avec de gros dialyseurs, il est possible d'y plonger un petit agitateur en verre à ailettes, afin d'assurer par une rotation lente un mouvement perpétuel du liquide; en changeant ainsi les surfaces à chaque instant, on accélère la dialyse.

On peut encore faire barboter un gaz inerte; par l'agitation ainsi communiquée au liquide, on aboutit également au renouvellement de la couche liquide en contact avec la paroi du dialyseur.

$3^{\circ}$ DiALyse a chaud et dialyse a FRoId. - Si on emploie l'eau chaude pour la dialyse, évidemment, on accentue la rapidité de celle-ci. Il est facile, en effet, avec un radiateur à gaz de faire cireuler de l'eau dans le dialyseur et de chauffer à un degré convenable pour que cette eau ait elle-même une température aux environs de $40^{\circ}$, sans grandes oscillations.

Avec des dialyses qui durent fort longtemps, il n'est guère possible d'utiliser de l'eau distillée; c'est de l'eau de la conduite dont on va se servir, qu'il s'agisse d'ailleurs de faire la dialyse à chaud ou la dialyse à froid. Cette eau est toujours légèrement calcaire ; cette considération, généralement sans grande importance, ne nous a pas arrêté dans nos recherches et ne nous a point fait modifier les conclusions auxquelles celles-ci pouvaient nous conduire. On a d'ailleurs la ressource de terminer la dialyse contre de l'eau distillée.

4. EAU " COURANTE " OU EAU " FIXE \#, - La dialyse sous l'eau courante est réalisée avec des vases que l'on trouve dans le commerce ou avec des dispositifs que l'on réalise facilement au laboratoire. Dans le bouchon qui supporte le tube en verre sur lequel est fixé le dialyseur en collodion sont percées deux ouvertures, l'une donnant accès à un tube allant jusqu'au fond du récipient, et l'autre permettant l'évacuation du surplus et s'arrêtant en-dessous du bouchon. Il est toujours facile de régler la vitesse de l'arrivée du courant d'eau de façon que le dialyseur plonge tout entier dans l'eau du flacon.

Quand on dialyse dans l'eau courante, on néglige de propos délibéré ce qui traverse la membrane. On ne peut évidemment s'intéresser qu'à ce qui reste dans le dialyseur et, à la fin de l'opération, on le comparera avec le liquide originel. Bien entendu, il faut tenir compte, - une fois pour toutes, nous faisons cette observation, - des variations de volume à l'intérieur du dialyseur.

Lorsqu'on veut étudier quantitativement et qualitativement ce qui se passe lors de la dialyse à travers la membrane, il faut évidemment employer ce que nous appelons l'eau fixe, c'est-à-dire ne pas entretenir de courant autour du dialyseur.

Le bouchon soutenant le dialyseur est plongé dans un flacon rempli 
d'eau distillée que l'on peut renouveler à intervalles choisis, afin de se rendre compte de la marche de la dialyse et de la vitesse du passage de tel ou tel élément considéré.

Au lieu de fixer le tube qui soutient le filtre en collodion dans un bouchon de liège, on peut l'entourer de plusieurs couches de coton de façon à le faire entrer à frottement doux dans le col du flacon.

La dialyse contre l'eau fixe a cet avantage, c'est qu'il devient possible d'opérer aseptiquement. On peut, en effet, stériliser le flacon rempli d'eau, muni au préalable de son dialyseur dont le tube qui le supporte aura été lui-même bouché par un tampon de coton. On peut même prévoir des dispositifs permettant de changer l'eau et de la remplacer par une autre eau également stérile.

(A suivre.)

\section{BIBLIOGRAPHIE ANALYTIQUE}

\section{$1^{\circ}$ JOURNAUX, REVUES, SOGIÉTÉS SAVANTES PRODUCTION, HYGIÈNE}

Friend lee mickle. Epreuve-type de I'Etat du Connecticut pour le sédiment du lait. Connecticut Health Bulletin, Vol. 42, No 2, février 1928, p. 27-30. (D'après les Public Heaith Repon is, 8 juin 1928).

L'auteur indique que les disques-types préparés d'après les méthodes-types d'analyse du lait de l'Association Américaine d'Hygiène Publique ont été trouvés inexacts au Connecticut. Les résultats de laboratoire de divers laboratoires officiels n'ont pas concordé avee les résultats des essais. Les méthodes-types sont aussi critiquées pour ne pas spécifier l'appareil particulier à employer dans l'essai. On indique aussi que les dimensions des particules de sédiment sur les différents types variaient beaucoup, et que la nature de sédiment employé dans divers laboratoires variait beaucoup plus de couleur qu'on ne l'avait précédemment pensé possible. Une photographie supplémentaire de disques a été adoptée (en supplément des

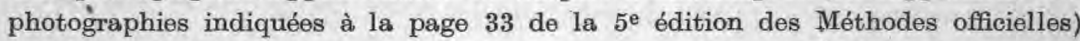
montrant $0,25,0,50,0,75$ et 1 milligramme de poussière par pinte ainsi que le modèle Wisconsin ou Lorenz de sédiment-témoin choisi comme type. Dans l'article est comprise la méthode exacte d'exécution de l'essai adoptée.

Coates (W. O.). - Réglementation du lait et des laiteries. Journal royal sanitary institute. Vol. 48, No 8, février 1928, p. 462-469. (D'a près les Public Health Reports, 8 juin 1928).

Cet article étudie le décret anglais sur le lait et les laiteries voté en 1926 et qu ${ }^{i}$ réglemente les installations de laiteries et leur contrôle par les autorités sanitaires.

C. divise le règlement comme suit: $1^{0}$ les deux autorités administratives; $2^{\circ}$ le personnel administratif, e'est-à-dire l'inspecteur vétérinaire, le médecin et l'inspecteur sanitaire; $3^{\circ}$ l'inspecteur sanitaire étant responsable de l'exécution de la plus grande partie du décret. Cette partie est divisée pour montrer l'importance 